\section{IMMUNOTHERAPEUTIC AND ANTIMETASTATIC ACTIVITY OF LTX-315 IN PRECLINICAL MODELS OF ICI-RESISTANT BREAST CANCER}

${ }^{1}$ Takahiro Yamazaki*, ${ }^{1}$ Erik Wennerberg, ${ }^{2}$ Michal Hensler, ${ }^{1}$ Aitziber Buqué Martinez, ${ }^{1}$ Jeffrey Kraynak, ${ }^{2}$ Jitka Fucikova, ${ }^{1}$ Xi Zhou, ${ }^{3}$ Baldur Sveinbjornsson, ${ }^{3}$ Oystein Rekdal, ${ }^{1}$ Sandra Demaria, 'Lorenzo Galluzzi. 'Weill Cornell Medical College, New York, NY, USA; ${ }^{2}$ Sotio, Prague, Czech Republic; ${ }^{3}$ Lytix Biopharma, Tromso, Norway

Background Oncolytic peptides are attractive tools for the development of novel anticancer regimens [1]. LTX-315 is a synthetic peptide with a marked capacity to elicit tumor-targeting immunity in preclinical cancer models [2]. Indeed, LTX-315 has been shown to elicit immunogenic cell death (ICD) in malignant cells [3, 4] and to deplete immunosuppressive cells such as CD4+CD25+FOXP3 + TREG cells and myeloid-derived suppressor cells (MDSCs) from the tumor microenvironment (TME) [5]. Accordingly, LTX-315 synergized with immunogenic chemotherapeutics or immune checkpoint inhibitors (ICIs) in preclinical tumor models [5, 6]. Moreover, recent findings from a Phase I clinical trial in patients with advanced solid tumors (NCT01986426) indicate that intratumoral LTX-315 is safe, clinically active, and elicits alterations in the TME that support the initiation of anticancer immunity [7, 8]. However, the dependency of LTX-315 therapeutic effects on the immune system in preclinical models of breast cancer has not been mechanistically investigated.

Methods We harnessed three distinct mouse models of ICIresistant breast cancer, namely hormone receptor (HR)-positive TS/A established and triple-negative breast cancer (TNBC) 4T1 cells established in immunocompetent syngeneic BALB/c mice, as well as medroxyprogesterone acetate (MPA, M)-initiated, 7,12-dimethylbenz[a]anthracene (DMBA, D)-driven mammary carcinomas evolving in $\mathrm{C} 57 \mathrm{BL} / 6$ mice to assess the immunotherapeutic effects of LTX-315 optionally combined with radiation therapy (RT), based on the primary tumor growth, metastatic dissemination and overall survival (depending on model). Multilesion models, rechallenge assays, antibody-mediated depletion experiments as well as experiments in Rag1-/mice were employed to elucidate the mechanistic involvement of the immune system.

Results In the multilesion TS/A models, intratumoral LTX-315 to one lesion combined with hypofractionated RT to another lesion resulted in superior systemic disease control as manifested by eradication of a $3 \mathrm{rd}$ untreated lesion in up to $50 \%$ of mice, which were protected from a subsequent rechallenge with living TS/A cells. In the single lesion 4T1 model, LTX315 mediated enable robust local and metastatic disease control, which could be enhanced (only locally) with RT and dependent on natural killer (NK) cells, but less so on T lymphocytes (as determined with anti-asialo GM1 antibodies and Rag1-/- hosts). In the M/D-driven model, LTX-315 considerably controlled the growth of primary tumors and delayed relapse, an effect that depended on NK cells (as demonstrated with anti-NK1.1 antibodies).

Conclusions LTX-315, alone and combined with RT, mediates robust immunotherapeutic effects in multiple models of ICIresistant breast cancer. Intriguingly, NK cells appear to be required for such effects, potentially linked to the emergence of immunological memory.

Acknowledgements We are indebted to Dr. Fred Miller (Karmanos Cancer Center, Detroit, MI) for the kind gift of 4T1 cells, as well as to Dr. Karsten A. Pilones (Weill Cornell Medicine, New York, NY) and Maria E. Rodriguez-Ruiz (University of Navarra, Pamplona, Spain) for help with clonogenic assays.
This work has been sponsored by a research grant by Lytix Biopharma (Oslo, Norway) to S.D. and L.G.

\section{REFERENCES}

1. Kepp, 0. et al. (2020) Oncolysis without viruses - inducing systemic anticancer immune responses with local therapies. Nat Rev Clin Oncol 17 (1), 49-64.

2. Vitale, I. et al. (2021) Targeting Cancer Heterogeneity with Immune Responses Driven by Oncolytic Peptides. Trends Cancer 7 (6), 557-572.

3. Eike, L.M. et al. (2015) The oncolytic peptide LTX-315 induces cell death and DAMP release by mitochondria distortion in human melanoma cells. Oncotarget 6 (33), 34910-23.

4. Zhou, H. et al. (2016) The oncolytic peptide LTX-315 triggers immunogenic cell death. Cell Death Dis 7 (3), e2134.

5. Yamazaki, T. et al. (2016) The oncolytic peptide LTX-315 overcomes resistance of cancers to immunotherapy with CTLA4 checkpoint blockade. Cell Death Differ 23 (6), 1004-15.

6. Camilio, K.A. et al. (2019) Combining the oncolytic peptide LTX-315 with doxorubicin demonstrates therapeutic potential in a triple-negative breast cancer model. Breast Cancer Res 21 (1), 9.

7. Jebsen, N.L. et al. (2019) Enhanced T-lymphocyte infiltration in a desmoid tumor of the thoracic wall in a young woman treated with intratumoral injections of the oncolytic peptide LTX-315: a case report. J Med Case Rep 13 (1), 177.

8. Spicer, J. et al. (2021) Safety, Antitumor Activity, and T-cell Responses in a DoseRanging Phase I Trial of the Oncolytic Peptide LTX-315 in Patients with Solid Tumors. Clin Cancer Res 27 (10), 2755-2763.

Ethics Approval This study was approved by Weill Cornell Medical College's Ethics Board; approval number 2015-0028, 2018-0002.

http://dx.doi.org/10.1136/jitc-2021-SITC2021.560 